\title{
EVALUACIÓN DE TRES NIVELES DE HARINA DEL FRUTO DE SIEMPREVIVA(Sempervivum tectorum) PARA EL CRECIMIENTO Y ENGORDE DE CUYES(Cavia porcellus)
}

\author{
EVALUATION OFTHREE LEVELS OF FLOUR THE FRUIT OF FOREVER \\ LIVING (Sempervivum tectorum) FOR GROWTH AND GUINEA PIG \\ FARMING (Cavia porcellus)
}

\author{
Juan Castro Cancino'; Rosario Telles Velásquez ${ }^{2}$
}

\begin{abstract}
RESUMEN
En el centro experimental de la granja de cuyes Liendo, hemos evaluado tres niveles de harina de fruto de siempreviva (Sempervivum tectorum) para el crecimiento ( 15 a 45 días) y engorde (60-69 dias) de cuyes (Cavia porcellus ). Se observó que en todos los casos (machos y hembras) los animales buscaron compensar la ingestión de nutrientes y energía con el mayor consumo de alimentos. No existen diferencias significativas entre densidades nutricionales para ganancia de peso $(p>0.001)$.

En la conversión alimenticia dentro de cada sexo tampoco se encontró diferencias importantes. Los rendimientos de carcasa se halla dentro de los rangos establecidos para esta especie. No haciendo diferencias significativas $(p>0.01)$ para el rendimiento de carcasa.

Al realizar la evaluación se ha podido apreciar que para el caso de los machos, los mayores beneficios económicos son alcanzados con el tratamiento T2 (108\%); sin embargo, para el caso de las hembras, con la densidad nutricional $(100 \%)$ de 2,58 Mcal EM/kg de alimento. Pero las mayores retribuciones económicas han sido logradas en los machos frente a las hembras, lo que implicaría que el engorde de machos es mucho más conveniente que el de las hembras.
\end{abstract}

Palabras claves: fruto de siempre viva, conversión alimenticia, rendimiento de carcasa, costo de producción.

\section{ABSTRACT}

In the experimental center Liendo guinea pig farm, we evaluated three levels of evergreen fruit flour (Sempervivum tectorum) for growth (15-45 days) and fattening (60-69 days) of guinea pigs (Cavia porcellus). It was observed that in all cases (males and females) animals sought compensate nutrient and energy intake with increased food intake. No significant differences between densities nutritional weight gain $(p<0.001)$.

Feed conversion within each sex also found important differences. The carcass yield is within ranges established for this species. Making no significant differences $(p>0.01)$ for carcass yield.

In conducting the evaluation it has been seen that in the case of males, the greatest economic benefits are achieved with treatment T2 (108\%), but in the case of females, the nutrient density (100\%) of $2.58 \mathrm{Mcal} \mathrm{ME} \mathrm{/} \mathrm{kg} \mathrm{feed.} \mathrm{But}$ the greatest economic returns have been achieved in males versus females, which would imply that the fattening of males is much more convenient than females.

Key words: polylepis rugulosa, Polylepis besseri, Phenotypic Characterization, Queñua.

\footnotetext{
Médico Veterinario y Zootecnista, Magíster en Administración y Gestión Empresarial. Facultad de Ciencias Agropecuarias de la UNJBG.

${ }^{2}$ Médico Veterinario y Zooctenista. Facultad de Ciencias Agropecuarias de la UNJBG.
} 


\section{INTRODUCCIÓN}

El cuy, como animal nativo de los andes, constituye una importante fuente de proteína para el poblador andino. Su relativa facilidad de crianza y su demanda local y regional en continuo incremento lo ponen en ventaja productiva frente a otras especies pecuarias (Moreno, 1989).

La mayoría de productores emplean en la alimentación de cuyes forraje verde y residuos de cosecha, a un precio comparativamente menor al de los alimentos balanceados. No obstante sus índices productivos, no alcanzan niveles satisfactorios. Al utilizar sólo forrajes y residuos de cosecha varía la cantidad de nutrientes que poseen estos alimentos y en algunos casos no son suficientes para satisfacer la demanda de cuyes (Laforé, 1999). Esta situación conlleva al desarrollo de nuevas estrategias de alimentación que permitan optimizar la productividad de la crianza del cuy.

La acentuada escasez y demanda de fuentes de proteína animal, permite al productor agropecuario iniciarse en la producción de animales menores con lo cual busca satisfacer la demanda a bajos costos de producción. La carne de cuy es de alto valor nutritivo, proteína $20.73 \%$, grasa $7.8 \%$, menor en comparación con otras especies $0.81 \%$, siendo su costo de producción bajo. Contribuye de esta manera a la seguridad alimentaria de la población rural y últimamente como un producto bandera con fines de exportación,(Rojas A, 1968).

En la región Tacna se viene criando actualmente 69620 cuyes de los cuales 44781 corresponden al distrito de Tacna (Chauca J, 1989).

\section{MATERIALES Y MÉTODOS:}

\subsection{Lugar de experimentación}

El presente trabajo de investigación se realizó en la granja Liendo, en el distrito de Calana, departamento de Tacna, ubicada a una altura de $848 \mathrm{msnm}$, a una latitud de $17^{\circ} 56^{\prime} 28^{\prime \prime}$ y a una longitud de $70^{\circ} 10^{\prime} 50^{\prime \prime}$ con una temperatura promedio de $17.7^{\circ} \mathrm{C}$ (Senamhi 2010).

\subsection{Animales experimentales}

Se emplearon 40 cuyes entre machos y hembras de la línea Perú, destetados con 12 días de edad promedio, procedentes de la granja Liendo. Los animales fueron identificados con aretes dentro de cada unidad experimental.

\subsection{Instalaciones y equipos}

La granja Liendo cuenta con una instalación de tipo galpón, con una longitud de $16 \mathrm{~m}$., $7.5 \mathrm{~m}$. de ancho y una altura de $2.5 \mathrm{~m}$, Las jaulas son de metal, de $2 \mathrm{~m}$ de largo por $1 \mathrm{~m}$. de ancho. Cada jaula fue limpiada, desinfectada con vanodine (desinfectante) y recibió cama nueva, antes de colocar a los animales. En las jaulas que recibían forraje se instaló una forrajera y una tarima hechas de malla, empleadas para el suministro y almacenamiento de forraje, respectivamente. Para el suministro de agua y el concentrado preparado se han empleado recipientes de arcilla de 0,75 litros de capacidad. El pesado de los animales, del alimento concentrado $\mathrm{y}$ al forraje verde se hizo empleando una balanza electrónica con $1 \mathrm{~g}$ de sensibilidad.

\subsection{Variables de estudio}

$$
\begin{aligned}
& \text { Independientes } \longrightarrow \text { Crianza de cuyes } \\
& \text { Dependientes } \longrightarrow \text { Alfalfa y concentrado } \\
& \text { Intervinientes } \longrightarrow \text { Manejo de alfalfa-Línea } \\
& \text { de Cuyes. }
\end{aligned}
$$

\subsection{Muestra}

En el experimento se usaron 40 cuyes destetados, sin distinción del sexo, de 12 días de edad y con un peso promedio de 260 gramos de peso vivo, todos de tipo 1, clínica y aparentemente sanos.

\subsection{Procedimiento de muestreo}

El procedimiento se realizó al azar, sin importar la talla y el sexo de los animales a experimentar.

\subsection{MÉTODOS DE EVALUACIÓN}

\subsubsection{Formación de las unidades experimentales de estudio.}

Para el experimento, las jaulas fueron asignadas de uno a cuatro. Allí se alojaron a 10 cuyes distribuidos al azar y subdivididos. Los tratamientos entre los cuatro grupos experimentales también fueron asignados al azar..

\subsubsection{Obtención de la harina del fruto de siempre viva (Sempervivum tectorum).}

Se recolectó frutos de la planta Siempreviva (Sempervivum tectorum)_de las zonas desérticas de la localidad de Tacna, las que, luego de ser secadas al sol, fueron transformadas en harina con una moledora de uso tradicional. Esta harina fue suministrada en la ración de los tres tratamientos de cuyes (Cavia porcellus). 


\subsubsection{Trabajo experimental}

Los grupos de cuyes recibieron raciones experimentales, una para cada grupo. Los alimentos proporcionados una vez al día y la cantidad usada fueron registradis. Los residuos de los alimentos se pesaron antes de proporcionar el alimento del día siguiente.

Los cuyes se pesaron cada 7 días, antes de proporcionar alimento nuevo a la semana. Los datos obtenidos se registraron en fichas especialmente elaboradas para este fin. Previa a la experimentación, los animales fueron desparasitados. Para el cálculo de la conversión alimenticia y el mérito económico se trabajaron con los datos obtenidos en el campo y según los precios de los alimentos adquiridos.

\subsubsection{Recolección de datos}

En el campo: La información de campo fue obtenida directamente con la evaluación de los cuyes, materia de la investigación. Asimismo, se tomó en cuenta las calidades y precios de los insumos utilizados.

En la biblioteca: Se usaron libros especializados, revistas científicas y las tesis de investigación que tuvieron información directamente al tema de estudio.

\subsubsection{Tratamientos}

La ración base fue formulada considerando las necesidades nutritivas de los cuyes. Se planificó un suplemento balanceado en función con un porcentaje de $0 \%$ para el tratamiento $\mathrm{T}_{0} ; 5 \%$ para el Tratamiento $\mathrm{T}_{1} ; 10 \%$ para el tratamiento $\mathrm{T}_{2}$ y $20 \%$ para el tratamiento $T_{s}$ de la harina del fruto de siempreviva (Sempervivum tectorum) más una cantidad de alfalfa que con el aumento de peso de los cuyes (Cavia porcellus) se incrementó.

La fórmula del balanceo de la ración fue a base de:

Tabla $\mathrm{N}^{\circ}$ 01: Balanceo de raciones.

\begin{tabular}{|c|c|c|c|c|c|c|c|c|}
\hline Insumos & $\begin{array}{l}\text { c.p. } \\
\%\end{array}$ & $\mathrm{~T}$ 。 & $\begin{array}{c}\text { c.p. } \\
\%\end{array}$ & $T_{\text {I }}$ & $\begin{array}{c}\text { c.p. } \\
\%\end{array}$ & $T_{2}$ & $\begin{array}{c}\text { c.p. } \\
\%\end{array}$ & $T$, \\
\hline Siempre viva & 0 & 0 & 0.6 & 5 & 1.27 & 10 & 2.5 & 20 \\
\hline Maíz & 1.7 & 20.8 & 1.5 & 15.8 & 0.9 & 10.8 & 0 & 0 \\
\hline Soya & 2.7 & 4.2 & 2 & 4.2 & 2 & 4.2 & 1.5 & 3.2 \\
\hline Soya integral & 7 & 18.8 & 7 & 18.8 & 7 & 18.8 & 7 & 18.8 \\
\hline Afrecho & 7.3 & 56.3 & 7.3 & 56.3 & 7 & 56 & 7 & 56 \\
\hline Sal & & 0.2 & & 0.2 & & 0.2 & & 0.3 \\
\hline Proapak 2 & & 0.1 & & 0.1 & & 0.1 & & 0.2 \\
\hline Total & 18 & 100. & 18.2 & 100.4 & 18.1 & 100.1 & 18 & 98.5 \\
\hline
\end{tabular}

Fuente: Propia

\section{Variables de respuesta:}

\section{Variable independiente}

Raciones Experimentales: Se evaluaron tres tratamientos, correspondientes a cuatro tipos de raciones:

- $\mathrm{T}_{0}=0 \%$ de harina del fruto de siempreviva (Sempervivum tectorum en el concentrado + $100 \%$ concentrado.

- $\mathrm{T}_{1}=5 \%$ de harina del fruto de siempreviva (Sempervivum tectorum en el concentrado $+95 \%$ concentrado $(5 \% \mathrm{VC}+95 \% \mathrm{~F})$.

- $\mathrm{T}_{\mathrm{a}}=10 \%$ de harina del fruto de siempreviva (Sempervivum tectorum en el concentrado $+90 \%$ concentrado $(10 \% \mathrm{VC}+90 \% \mathrm{~F})$.

- $\mathrm{T}_{3}=20 \%$ de harina del fruto de siempreviva (Sempervivum tectorum en el concentrado $+80 \%$ de concentrado $(20 \% \mathrm{VC}+80 \% \mathrm{~F})$.

\section{Variable dependiente}

- Consumo de alimento y materia seca.

- Ganancia diaria de peso.

- Conversión alimenticia.

- Rendimiento y calidad de carcasa.

- Mérito económico.

Diseño experimental: El Modelo aditivo lineal fue el siguiente:

Donde:

$$
\mathrm{Yij}=\mu+\mathrm{Ti}+\square \mathrm{ij}
$$

$\mathrm{Yij}=$ Peso vivo del tratamiento $\mathrm{i}$, unidad $\mathrm{j}$

$\mu=$ Media poblacional

$\mathrm{Ti}=$ Efecto del $\mathrm{i}$-esimo tratamiento, raciones experimentales $(\mathrm{i}=1,2,3,4)$.

$\mathrm{j}=1,2,3,4$, repeticiones

$\square \mathrm{ij}=$ Efecto de la j-esimo observación del ieo

si tratamiento(error experimental).

Prueba de significancia: Para la comparación de medias y comprobar las diferencias reales entre tratamientos se utilizó la prueba de rango múltiple de Duncan (Calzada, 1982).

\subsubsection{Manejo experimental}

Se utilizó alfalfa verde de la variedad $\mathrm{WL}-625$, ofrecida a una frecuencia de dos veces por día (a las 8:00 y 16:00 horas). La alfalfa verde se compró tres veces por semana, cuando esta llegó al galpón se desinfectó y pesó la ración por poza, para dos o tres 
días, considerando un consumo diario individual equivalente al $4.0 \%$ del peso vivo animal, el cual fue almacenado en la tarima de cada jaula, desde donde se suministró las tres raciones diarias. El día del pesaje del forraje también se tomó una muestra para estimación del contenido de materia seca del alimento administrado. $\mathrm{El}$ alimento rechazado se pesó semanalmente y también se tomó una muestra para estimación de materia seca. Se realizó el análisis proximal del forraje muestreado a lo largo del experimento, con la finalidad de monitorear la calidad del forraje ofrecido. La harina del fruto de siempreviva (Sempervivum tectorum) para la suministración en la ración del cuy se ofreció a discreción, una vez por día (8:00 horas), a los tratamientos del T1, T2, y T3, empleando un recipiente de arcilla.

- Determinar la eficiencia alimenticia durante el crecimiento y engorde de cuyes (Cavia porcellus).

- Determinar la velocidad de crecimiento

\subsubsection{Variables evaluadas}

Ganancia de peso: Se tomaron pesos individuales semanales a todos los animales, desde el inicio del experimento hasta que alcancen el kilo de peso vivo, peso con el que finalizaban su permanencia en el experimento. Con esa información se calculó la ganancia de peso diaria individual, entre la segunda y octava semana, momento en que un primer grupo de animales alcanzó el kilo de peso vivo, es decir toda las unidades experimentales estuvieron completas sólo hasta la octava semana.

Índice de conversión alimenticia: $\mathrm{El}$ índice de conversión alimenticia resultó de dividir el consumo total de materia seca entre la ganancia de peso total en cada unidad experimental.

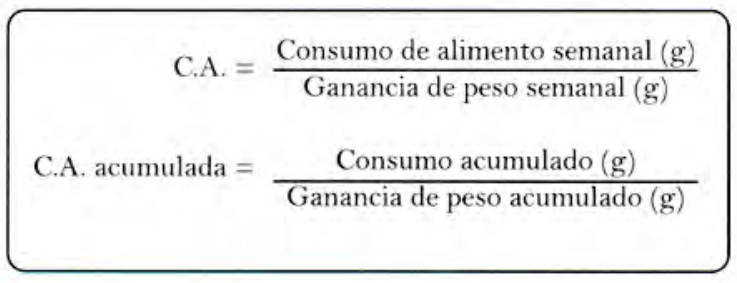

Eficiencia alimenticia: Es la cantidad de gramos de alimento ingerido para producir un kilogramo de carne.

$\mathrm{EA}=$ consumo de alimento $\mathrm{Kg}$ de carne
Rendimiento de carcasa: Para el rendimiento de carcasa, los animales beneficiados fueron sometidos a un ayuno de 24 horas antes del beneficio. La carcasa incluye piel, cabeza, patitas y órganos (corazón, pulmón, hígado, bazo y riñón) y para la evaluación de este parámetro se utilizaron 2 animales por tratamiento.

Evaluación económica: Se estimó el costo de producción según las condiciones del galpón demostrativo, dicha información se utilizó para conocer el valor porcentual que representa el costo de alimentación de cada tratamiento respecto al costo de producción total. Con estos datos se estimó el costo de producción conociendo sólo el costo de alimentación para cada unidad experimental.

\section{RESULTADOS}

Tabla $\mathrm{N}^{\circ}$ 02: Consumo de alimento.

\begin{tabular}{|c|c|c|c|c|c|}
\hline \multirow{2}{*}{ TRATAMIENTO } & \multirow{2}{*}{$\begin{array}{c}\text { DENSIDAD } \\
\text { NUTRICIONAL }\end{array}$} & \multicolumn{2}{|c|}{$\begin{array}{l}\text { CONSUMO } \\
\text { MACHOS }\end{array}$} & \multicolumn{2}{|c|}{$\begin{array}{l}\text { CONSUMO } \\
\text { HEMBRAS }\end{array}$} \\
\hline & & $\mathrm{g} /$ día & Total, g & $\mathrm{g} /$ dia & Total, g \\
\hline $\mathrm{Tl}$ & 2.58 & 54.60 & $2675.38^{b}$ & 50.30 & $2464.75^{\circ}$ \\
\hline $\mathrm{T} 2$ & g.48 & 56.94 & $2790.38^{b}$ & 53.22 & $2607.88^{b}$ \\
\hline Ts & 2.38 & 59.64 & $2922.59=$ & 55.65 & $2726.75=$ \\
\hline
\end{tabular}

Fuente: Propia

En la Tabla $\mathrm{N}^{\circ} \mathrm{O} 2$, se observa los datos acerca de la ingestión de nutrientes. En todos los casos los animales buscaron compensar la ingestión de nutrientes y energía con el mayor consumo de alimentos.

Tabla N $^{\circ}$ 03: Ganancia de peso.

\begin{tabular}{|c|c|}
\hline TRATAMIENTOS & $\begin{array}{c}\text { GANANCIA } \\
\text { DE PESO(g) }\end{array}$ \\
\hline T1 & 399.44 \\
\hline T2 & 460.75 \\
\hline T 3 & 380.19 \\
\hline TESTIGO & 372.13 \\
\hline
\end{tabular}

Fuente: Propia

En la Tabla $\mathrm{N}^{\circ}$ 03, no se encontraron diferencias significativas entre densidades nutricionales para ganancia de $\operatorname{peso}(\mathrm{p}>0.001)$, lo que implicaría que las variaciones en las densidades nutricionales no afectaron la tasa de crecimiento de los cuyes, que estaría determinado por el mayor consumo de alimento realizado en los tratamientos con menores densidades nutricionales, con el objetivo de cubrir la demanda de nutrientes y principalmente de energía, sin embargo la NRC (1978) reporta mejor 
repuesta en ganancia de peso y eficiencia alimenticia con las dietas de mayor densidad energética.

Tabla $N^{\circ}$ 04: Conversión alimenticia

\begin{tabular}{|c|c|c|c|}
\hline TRATAMIENTO & $\begin{array}{c}\text { DENSIDAD } \\
\text { NUTRICIONAL }\end{array}$ & $\begin{array}{c}\text { CONSUMO } \\
\text { MACHOS }\end{array}$ & $\begin{array}{c}\text { CONSUMO } \\
\text { HEMBRAS }\end{array}$ \\
\hline $\mathrm{T} 1$ & 2.58 & $6.08^{\mathrm{b}}$ & $6.94^{\mathrm{a}}$ \\
\hline $\mathrm{T} 2$ & 2.48 & $5.08^{\mathrm{b}}$ & $7.26^{\mathrm{c}}$ \\
\hline $\mathrm{T} 3$ & 2.38 & $5.94^{\mathrm{a}}$ & $9.56^{\mathrm{a}}$ \\
\hline
\end{tabular}

Fuente: Propia

En la Tabla $\mathrm{N}^{\circ} \mathrm{O4}$, al realizar el análisis de la conversion alimenticia, por cada sexo y por densidad nutricional, no se encontraron diferencias significativas para las tres densidades nutricionales $(\mathrm{p}<0,01)$.

Tabla N ${ }^{\circ} 05:$ Rendimiento de carcasa

\begin{tabular}{|c|c|c|c|c|}
\hline TurraMmarro & 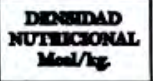 & $\underset{(1000}{1000}$ & $\begin{array}{c}\text { moo } \\
\text { cancas } \\
\theta\end{array}$ & $\begin{array}{c}\text { nonmorro } \\
\text { (x) }\end{array}$ \\
\hline T1 & 2.58 & $911.5^{2}$ & 595.28 = & $65.75^{2}$ \\
\hline Te & 2.48 & 951.0" & 618.47 " & 68.08 * \\
\hline Ts & 2.38 & $847.5=$ & $610.74^{2}$ & 67.01 a \\
\hline
\end{tabular}

Fuente: Propia

En la Tabla $\mathrm{N}^{\circ} \mathrm{05}, \mathrm{los}$ rendimientos de carcasa registrados se encuentran dentro de los rangos establecidos para esta especie y han sido evaluados por el INIA (2005).

$\mathrm{Al}$ analizar los resultados, no se encontraron diferencias significativos entre densidades nutricionales para el rendimiento y porcentaje de carcasa.

En la Tabla $\mathrm{N}^{\circ} 06$, la retribución económica es mejor para el tratamiento 2, frente a los otros tratamientos. En ambos casos las mayores retribuciones económicas son logradas en todas las densidades nutricionales empleadas en los machos frente a las hembras, lo que implicaría que el engorde de machos es mucho más conveniente frente a las hembras.

\section{DISCUSIÓN}

Los pesos iniciales no presentan diferencias estadísticamente significativas, debido a que en la investigación se trabajó con animales destetados, provenientes de un mismo galpón.

Freire G (2004) realizó una evaluación de diferentes niveles de proteínas (14,17 y 20\%) en raciones para crecimiento y engorde de cuyes destetados precozmente. Utilizó cuyes con un peso promedio de $247 \mathrm{~g}$ y 10 días de edad, para evidenciar el efecto de los pesos de los animales en la etapa de recría. Además, Pazmiño D. (2005), al evaluar los diferentes niveles de cáscara de maracuyá como subproducto no tradicional en la alimentación de cuyes, utilizó animales con un peso inicial promedio $298 \mathrm{~g}$; estos valores son inferiores a los resultados actualmente obtenidos. Probablemente el autor antes mencionado utilizó cuyes en edad de 15 días, razón por la cual los pesos en la etapa de recría varíe altamente con nuestros resultados.

$\mathrm{Al}$ observar los resultados, respecto a la ganancia de peso, se registraron diferencias estadísticamente significativas. Esto se debe a que mientras disminuye la relación de energía proteína, se incrementa la ganancia de peso.

Tabla $\mathrm{N}^{\circ}$ 06: Mérito económico.

\begin{tabular}{|c|c|c|c|c|c|c|c|c|c|}
\hline \multirow{2}{*}{ VARIABLE } & \multicolumn{3}{|c|}{ GENERAL } & \multicolumn{3}{|c|}{ MACHOS } & \multicolumn{3}{|c|}{ HEMBRAS } \\
\hline & $\mathrm{T} 1$ & $\mathrm{~T} 2$ & Ts & $\mathrm{T}_{1}$ & $\mathrm{~T} 2$ & $\mathrm{Ts}$ & $\mathrm{T}_{1}$ & $\mathrm{~T} 2$ & Ts \\
\hline \multirow{4}{*}{$\begin{array}{l}\text { INGRESOS } \\
\text { Peso vivo, Kg. } \\
\text { Precio por Kg. P.V., S/. } \\
\text { Ingreso bruto/cuy, S/. }\end{array}$} & & & & & & & & & \\
\hline & 0.889 & 0.910 & 0.827 & 0.922 & 1.013 & 0.921 & 0.845 & 0.807 & 0.744 \\
\hline & 12.00 & 12.00 & 12.00 & 12.00 & 12.00 & 12.00 & 12.00 & 12.00 & 12.00 \\
\hline & 10.60 & 10.92 & 9.92 & 11.060 & 12.156 & 11.052 & 10,140 & 9.684 & 8.928 \\
\hline \multirow{4}{*}{$\begin{array}{l}\text { EGRESOS } \\
\text { Cantidad de alimento, } \mathrm{Kg} \text {. } \\
\text { Precio alimento, S/. Kg. } \\
\text { Costo alimentación, S/. }\end{array}$} & & & & & & & & & \\
\hline & 2.57 & 2.70 & 2.82 & 4.827 & 2.790 & 2.922 & 2.465 & 2.608 & 2.727 \\
\hline & 0.87 & 0.84 & 0.78 & 2.50 & 0.84 & 0.78 & 0.87 & 0.84 & 0.78 \\
\hline & 2.232 & 2.271 & 2.211 & 2.327 & 2.344 & 2.279 & 2.144 & 2.191 & 2.127 \\
\hline \multirow{3}{*}{$\begin{array}{l}\text { COSTO TOTAL } \\
\text { Costo por cuy, S/. } \\
\text { Costo alimentación, S/. }\end{array}$} & 4.732 & 4.771 & 4.711 & 4.827 & 4.844 & 4.779 & 4.644 & 4.691 & 4.627 \\
\hline & 2.50 & 2.50 & 2.50 & 2.50 & 2.50 & 2.50 & 2.50 & 2.50 & 2.50 \\
\hline & 2232 & 2.271 & 2.271 & 2.327 & 2.344 & 2.279 & 2.144 & 2.191 & 2.127 \\
\hline \multirow{4}{*}{$\begin{array}{l}\text { RETRIBUCIÓN } \\
\text { Beneficio por cuy, S/. } \\
\text { Por Kg. De peso vivo, Kg. } \\
\text { Porcentaje }\end{array}$} & & & & & & & & & \\
\hline & 5.868 & 6.149 & 5.209 & 6.233 & 7.312 & 6.273 & 5.496 & 4.993 & 4.301 \\
\hline & 6.645 & 6.757 & 6.299 & 6.760 & 7.218 & 6.811 & 6.504 & 6.187 & 5.781 \\
\hline & 100 & 102 & 95 & 100 & 108 & 101 & 100 & 95 & 89 \\
\hline
\end{tabular}

Fuente: Propia 
Pazmiño D. (2005), al evaluar los diferentes niveles de tratamiento con sub productos no tradicionales en la alimentación de cuyes, encontró valores que resultan mayores a los obtenidos en nuestro trabajo, esto probablemente se debe a la influencia y aceptabilidad de dichos sub-productos utilizados como alimentación.

Del análisis relacionado a la conversión alimenticia en la etapa de recría y engorde, se observó diferencias estadísticamente significativas para la conversión alimenticia.

Los valores de las conversiones alimenticias encontrados guardan relación con los reportados por Cabay L. (2000), al evaluar la alimentación de forrajes, más el efecto de tres niveles de semillas de zapallo.

\section{CONCLUSIONES}

- En cuanto a la ingestión de nutrientes, se observó que en todos los casos los animales buscaron compensar la ingestión de nutrientes y energía con el mayor consumo de alimentos.

- En el análisis de los resultados, no se encontró diferencias significativas entre densidades nutricionales para ganancia de peso $(p>0.001)$, lo que implicaría que las variaciones en las densidades nutricionales no afecta la tasa de crecimiento de los cuyes, que estaría determinado por el mayor consumo de alimento realizado en los tratamientos con menores densidades nutricionales con el objetivo de cubrir la demanda de nutrientes.

- La conversión alimenticia por sexo y por densidad nutricional no encontró diferencias significativas entre machos y tampoco entre hembras, para las tres densidades nutricionales.

- Los rendimientos de carcasa registrados se encuentran dentro de los rangos establecidos para esta especie y que han sido evaluados por el INIA(2005). No habiendo diferencias significativas entre densidades nutricionales para el rendimiento y porcentaje de carcasa.

- En términos generales, se aprecia que la retribución económica es mejor en el tratamiento 2 , frente a los otros y que corresponde a la densidad de 2,48 Mcal $\mathrm{EM} / \mathrm{kg}$ de alimento.

\section{RECOMENDACIONES}

Las recomendaciones que se pueden emitir en base a las respuestas obtenidas en el presente trabajo serían las siguientes:

- Buscar alternativas de formulación de los balanceados con la utilización de otros insumos no tradicionales para comprobar si estos son digeribles por los cuyes y que sean aprovechados a través del desdoblamiento en la actividad cecotrófica que poseen y que permitan la reutilización del nitrógeno proteico y no proteico que no alcanzó a ser digerido en el intestino.

- Continuar con el estudio de utilización de insumos no tradicionales por cuanto en el presente trabajo se obtuvo buenos resultados para beneficiar a los criadores y sirva de base científica para otras posteriores investigaciones.

\section{REFERENCIAS BIBLIOGRÁFICAS}

Aliaga R. (1996). Crianza de cuyes. Instituto Nacional de Investigación Agraria. Dirección General de Transferencia Tecnológica. LimaPerú.

Álvarez, J. (1999). Evaluación de dos niveles de energía y tres de proteína en el crecimiento de cuyes destetados, con raciones en base a alfalfa, maíz, afrecho, soya y harina de pescado. Tesis del Programa Profesional de Medicina Veterinaria y Zootecnia de la UCSM. Arequipa-Perú.

Allendez Z. Y Canas R. (1985). Utilización de Subproductos de Matadero en Alimentación Animal-- Tesis Facultad de Agronomia Universidad Catolica de Chile.

Arroyo Barreto Oscar. (1986). Avances de Investigación sobre Cuyes en el Perú.- Serie Informes Técnicos del Instituto Nacional de Investigación y Promoción Agropecuaria de lima.

Arispe T. (1999). Efecto del uso de cinco niveles de aceite acidulado de pescado sobre el comportamiento productivo de cuyes en crecimiento en la Campiña de Arequipa. Tesis del Programa Profesional de Medicina Veterinaria y Zootecnia de la UCSM. Arequipa-Perú. 
Cerna, M.A. (1997). Evaluación de cuatro niveles de residuos de cervecería seco en el crecimiento-engorde de cuyes. Tesis de la Facultad de Zootecnia de la UNA - LM. LimaPeru.

Espinoza, F. y Morillo, Y. (2005). Uso de la harina de leucaena (Leucaena leucocephala) en la alimentación de corderos post-destete en la época seca. Revista de la Facultad Agronomía. (LUZ)Aragua-Venezuela. 41-51 pag.

\section{Correspondencia:}

Juan Castro Cancino

Ciudad Universitaria fundo "Los Granados"

Av. Miraflores s/n Tacna - Perú

Rosario Telles Velásquez

Ciudad Universitaria fundo "Los Granados"

Av. Miraflores s/n Tacna - Perú

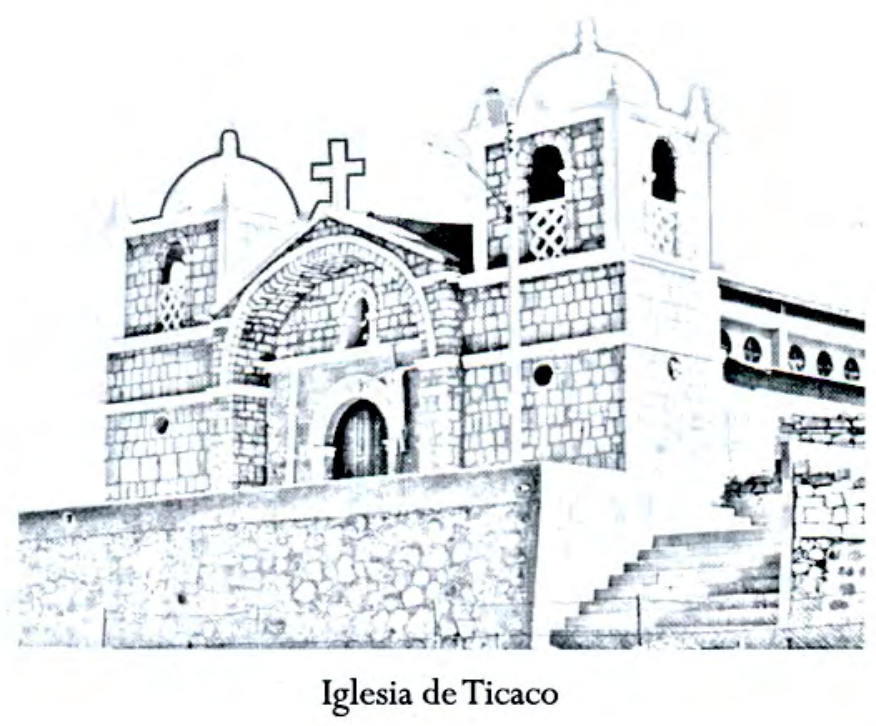

